\title{
LA COBERTURA DE LA CIENCIA EN LOS NOTICIEROS \\ COLOMBIANOS: DEL ANÁLISIS DE RESULTADOS A LAS REFLEXIONES METODOLÓGICAS PARA SU INVESTIGACIÓN'
}

\begin{abstract}
RESUMEN: Basados en los resultados de un estudio de cobertura de ciencia y tecnología en el noticiero ${ }^{2}$ Noticias Caracol de Colombia, realizado entre octubre del 2009 y marzo del 2010, nos preguntamos críticamente por la marcada tendencia que esta investigación indica, en diálogo con pesquisas similares, sobre lo que parece ser una baja presencia de estas temáticas en los medios informativos latinoamericanos. Inicialmente discutimos la metodología utilizada en este estudio y otros similares. Luego nos centramos en las noticias sobre agua y medio ambiente, que son las más recurrentes en este estudio, para finalmente preguntarnos por los contextos de riesgo que éstas tácitamente evocan y que están atravesados por concepciones particulares de la ciencia y la tecnología.

Palabras clave: Cobertura de ciencia y tecnología, telediarios, recurso hídrico, riesgos.
\end{abstract}

\section{SCIENCE COVERAGE IN COLOMBIAN NEWCASTS: FROM THE ANALYSIS OF RESULTS TO METHODOLOGICAL REFLECTIONS FOR ITS INVESTIGATION}

ABSTRACT: Based on theresults of a study on science and technology coverage in the Colombiantelevision newscast Noticias Caracol,between October 2009 and March 2010, we asked, from a critical perspective, thereasons for the low presence of these themes in Latin American news shown inthat research, along with similar studies. Initially, we discussed themethodology of the study and similar ones. Subsequently, we focused on waterand environment news, because these themes were the most frequent. Finally, wediscussed the risk contexts that these newscasts tacitly evoke and that are permeatedwith particular conceptions of science and technology.

Keywords: Science and technology coverage, news programs, waterresources, risks.
Tania Arboleda Castrillón*

Daniel Hermelin**

Tania Pérez Bustos***

*Profesora del Departamento de Comunicación Social, Facultad de Comunicación y Lenguaje de la Pontificia Universidad Javeriana

Bogotá. Integrante del grupo de investigación Comunicación Medios y Cultura del mismo departamento. E-mail: tarboleda@javeriana.edu.co

* * Profesor del Programa de

Comunicación Social y del

Departamento de Humanidades de la Universidad EAFIT (Medellín). Integrante del grupo de investigación en Política y Lenguaje del mismo departamento.

E-mail: dhermeli@eafit.edu.co

***Profesora del Departamento de Antropología, Facultad de Ciencias Sociales de la Pontificia Universidad Javeriana, Bogotá. Integrante del grupo de investigación Estudios sobre identidades y prácticas de poder del mismo departamento.

E-mail: tpbustos@gmail.com 


\section{INTRODUCCIÓN}

En el marco del trabajo realizado en la Red Iberoamericana de Monitoreo y Capacitación en Periodismo Cientifico, emprendimos, entre 2009 y 2010, un rastreo de noticias en ciencia y tecnología en telediarios de diferentes países de América Latina. Para Colombia el estudio se enfocó en uno de los noticieros de mayor audiencia en el país, Noticias Caracol de las 7:00pm. Este estudio buscó dar cuenta de la presencia o no de ciencia y tecnología en este espacio informativo, y confirmó lo ya encontrado en estudios similares: que la ciencia y la tecnología suscitan poco interés en el periodismo latinoamericano. Quisimos asumir estos resultados con beneficio de inventario, preguntándonos por aquello que ciertos abordajes analíticos sobre la cobertura en ciencia y tecnología no dejan ver en contextos periféricos como el colombiano, pues se centran en la ciencia como un hecho dado, en muchos casos como hallazgo, y no como una construcción social, dejando de lado sucesos, que si bien no están enmarcados como de carácter científico o tecnológico, tácitamente aluden a estas cuestiones.

Siguiendo la propuesta de Haraway (1995) sobre el privilegio de las perspectivas parciales y la importancia de evidenciar aquellos supuestos que soportan la investigación, pero que en ocasiones son invisibilizados por la investigación misma, en este artículo reflexionamos sobre estos puntos ciegos en relación con la presencia de la ciencia y la tecnología en este noticiero colombiano, en particular. Iniciamos este ejercicio situando esta reflexión en el marco de las investigaciones sobre cobertura de ciencia y tecnología en medios que se han realizado en la región, para luego presentar la metodología que sirvió como base para el estudio mencionado y las tendencias encontradas; en ambos casos hacemos énfasis en los puntos ciegos que estos ejercicios tienen. Cerramos el artículo con unas consideraciones finales para futuros estudios sobre estos temas.

\section{ANTECEDENTES}

En el marco de aquellas visiones que promueven la necesidad de que la ciencia sea parte relevante en las decisiones de la vida cotidiana, un número considerable de estudios han buscado dar cuenta de la cobertura de la ciencia y la tecnología, particularmente en la prensa escrita en América Latina. La mayoría son estudios comparativos en los que se selecciona un corpus de noticias de los principales periódicos de cada país, para luego realizar análisis de contenido de la información obtenida en los rastreos. Estos trabajos tienen el propósito de proporcionarle información a la política pública de apoyo al fomento de la cultura científica a través de los medios de comunicación, o de proporcionar lecturas críticas y constructivas sobre el ejercicio del periodismo y la divulgación científica a los comunicadores que cubren estos temas y a los tomadores de decisión de los medios masivos escritos (BUSTOS-MORA, 2004; PARODI, FERRARI, 
2007; AMORIM, MASSARANI, 2008; MASSARANI, BUYS, 2007). Entre otras conclusiones que salen de estos estudios, se plantea que el género periodístico que predomina es la noticia, con una mayoría de fuentes de información masculinas, que el espacio otorgado a la información científica y tecnológica en la mayoría de los casos es bajo, sobre todo en los sucesos locales, así como que la tendencia a cubrir controversias es baja, evidenciando la disposición insuficiente de los medios para desarrollar estas temáticas ${ }^{3}$.

Además se observa cierta pasividad en el rol del periodista a la hora de explorar estos temas en el ámbito local, con una fuerte preferencia por citar agencias informativas y de Internet con una actitud poco crítica, lo cual provoca la homogeneización de la información sobre estos temas. Algunos estudios deducen y cuestionan con ello, el contacto limitado que los periodistas tienen con la actividad científica nacional y un escaso hábito investigativo (BUSTOS-MORA, 2004, p. 227); y otros plantean que esto es debido a

\footnotetext{
la falta de tradición cultural de los científicos locales de conceder rápidamente una entrevista -en contraposición al hábito de los investigadores norteamericanos de responder rápidamente a las preguntas de un periodista de cualquier nacionalidad- [lo cual] también estimula el uso de material proporcionado por esos servicios, provocando que un mismo tema sea publicado en varios periódicos del mundo. (AMORIM, MASSARANI, 2008, p.83).
}

Por otra parte, varios estudios concluyen que la información periodística sobre ciencia y tecnología se concentra en los resultados positivos de estos desarrollos, sin dar cuenta de los procesos de investigación que subyacen a estos resultados lo que contribuye a sacralizar la ciencia; sabiendo que "los propósitos últimos de algunos científicos no necesariamente, ni en todos los casos constituyen en sí mismos objetivos altamente loables y se orientan positivamente en la búsqueda de la verdad" (PARODI, FERRARI, 2007, p. 365). Por lo demás, las temáticas de salud y medio ambiente son las que aparecen como más ampliamente cubiertas, algo similar a lo que ocurre en el presente estudio como se discutirá más adelante.

En términos de las investigaciones sobre la cobertura de la ciencia en los medios masivos colombianos los resultados no distan mucho de lo encontrado en los estudios mencionados anteriormente. Rozo (2006), por ejemplo, al preguntarse por el tipo de cultura científica y cultura tecnológica transmitido por el periódico El Tiempo $o^{4}$ entre 1995 y 2004, plantea que el "unifuentismo" característico de la cobertura de estos temas en ese medio es más que llamativo, teniendo en cuenta que el periodismo habría de contribuir a generar capacidad de análisis y crítica de parte de la ciudadanía; esto a su vez constituye una condición de la apropiación social de la ciencia y la tecnología. Los textos analizados contribuyen a generar una imagen de la ciencia y la tecnología asociada al desarrollo empresarial e industrial, con tecnologías producidas e importadas del primer mundo. Por el lugar que ocupa este tipo de información en el periódico, se observa un interés económico del medio en relación con la misma, ello también sugiere "una visión separada de 
asuntos y aspectos que tienen consecuencias, implicaciones y efectos directos con diversos tópicos de la vida nacional" (2006, p.18).

Por último, encontramos estudios que abordan la pregunta por la información periodística en general y generan datos relevantes sobre la cobertura de ciencia y tecnología. Este es el caso de ¿Qué es noticia?, cuyo propósito era "descubrir esas imágenes de país que pasan por los medios a partir de sus relaciones, tanto con quienes las producen (los periodistas), como con quienes las consumen (los públicos lectores, radioyentes y televidentes)" (BONILLA, CADAVID, 2004, p.19). Entre otras conclusiones relevantes sobre las agendas periodísticas y las agendas ciudadanas en relación a los temas de ciencia y tecnología, el estudio determinó que los asuntos de salud, ciencia y medio ambiente rara vez logran ubicarse en posiciones prioritarias, ya sea en términos espaciales (primera página) o en temporales (titulares). Señala que en este tipo de información también predomina el "unifuentismo" o la falta de fuentes en los tres medios principales del país; en más de la mitad de los casos la fuente es oficial y en una cuarta parte son especialistas o académicos. Frente a la pregunta por las modalidades informativas que predominan en las agendas de los medios a la hora de informar sobre estos temas, se encontró que el 49\% de la información se presenta en forma de resultados, el $23 \%$ en forma de declaraciones y el $18 \%$ en forma de controversias.

Un estudio sobre la estructura, cobertura y contenidos de noticieros de televisión en Colombia (GÓMEZ-GIRALDO et al., 2010), revela datos muy similares a las conclusiones ya mencionadas: la agenda de los noticieros colombianos le otorga un $2 \%$ de relevancia a los temas de ciencia y tecnología, encuentra que las informaciones sobre ciencia y tecnología en su mayoría son noticias con un solo punto de vista que ponen énfasis en la descripción e información, e inscriben su importancia en el plano internacional. En términos del encuadre de la noticia, la mayoría de ellas ponen énfasis en la descripción e información (86\%) y en menor medida en el interés humano (9\%).

La mayoría de estos resultados refuerzan la concepción difusionista de la comunicación pública de la ciencia y la tecnología en el sentido de que contribuyen a definir los medios como "un canal designado para transmitir nociones científicas, que a menudo es incapaz de llevar a cabo esta tarea satisfactoriamente por falta de competencias y/o por la predominancia de otras prioridades (por ejemplo, intereses comerciales)" (BUCCHI, 2008, p. 58). Sin embargo, el estudio de Amorim y Massarani (2008) intenta romper esta visión estereotipada de los medios y los periodistas con lo cual hace parte del conjunto de estudios sobre la cobertura de ciencia y tecnología en medios que, según Bucchi, abogan por "una mayor precisión, una interacción más cercana entre periodistas y fuentes especializadas, y en general, por esfuerzos para minimizar los elementos que causan 'molestia' en la comunicación entre científicos y el público en general, que de otra manera sería más “directa” (2008, p.59). 


\section{APUNTES SOBRE LA METODOLOGÍA: ÉNFASIS, PUNTOS CIEGOS Y POSIBILIDADES}

En líneas metodológicas similares a las utilizadas por los estudios referidos arriba, para el rastreo de información sobre ciencia y tecnología en el telediario colombiano Noticias Caracol, sobre el que se basa este artículo, se hizo uso de un protocolo de análisis de contenido adaptado a partir de uno que se ha aplicado principalmente con prensa escrita en el ámbito latinoamericano (MASSARANI, et. al., 2008; MASSRANI, BUYS, 2007; MASSARANI, et. al., 2005). Estos estudios, por su parte, se han inspirado principalmente en el trabajo de Bauer et al. (1993) sobre la cobertura de ciencia y tecnología en contextos anglosajones, y también toman en cuenta algunas investigaciones de carácter comparativo sobre ciencia en la televisión entre países llamados desarrollados (PETERS et. al., 2008; PELLECHIA, 1997; CLARK, ILLMAN, 2006).

En el caso concreto del estudio colombiano se analizó la cobertura de ciencia y tecnología en Noticias Caracol de las $7 \mathrm{pm}^{5}$. El período de análisis fue de 6 meses (octubre del 2009 a marzo del 2010) y se basó en una muestra que se consolidó siguiendo la metodología de la "semana construida", uno de los métodos de muestreo que han demostrado mayor confiabilidad en el terreno del análisis de contenido en los medios de comunicación (STEMPEL, 1989; KRIPPENDORFF, 1990). La muestra equivale a 6 "semanas construidas" (de lunes a domingo), una por cada mes de la muestra, es decir que analizamos 42 programas.

La selección de las piezas informativas que conforman la muestra se hizo aplicando un protocolo de análisis con criterios cerrados de selección, para identificar la presencia de temas de ciencia y tecnología en cada uno de estos 42 programas. Así, el instrumento permitió identificar cierto tipo de información, a priori, más común en el periodismo científico, dándole prioridad a las notas en las que aparecen resultados de investigación, investigadores, instituciones de investigación, políticas de ciencia y tecnología y comunicación pública de estos temas. En este sentido, no se incluyeron reportajes sobre salud pública, medio ambiente o tecnología, a no ser que en éstos aparecieran científicos para explicar resultados de investigaciones, nuevos desarrollos en estos temas o datos relacionados con publicaciones científicas. En términos generales, un protocolo como éste, con sus respectivos criterios de inclusión, tiende a privilegiar el seguimiento al manejo que hacen los medios de los halla ggos cientificos ${ }^{6}$, lo que, por su parte, tiene como principal punto ciego la ausencia de noticias que refieren a ciencia y tecnología de modos más tácitos; principalmente porque se relacionan con problemáticas que están configuradas transversalmente por la interacción entre ciencia, tecnología y sociedad, como es el caso de los desastres naturales o de la gestión del agua.

Ahora bien, aunque los criterios de inclusión utilizados eran bastante cerrados, el protocolo dejó abierta la posibilidad de incluir noticas que en general se refirieran a temáticas específicas, definidas éstas por los intereses investigativos de las y los integrantes de la Red Iberoamericana de Monitoreo y Capacitación en Periodismo Cientifico. Así, el protocolo permitió que se incluyeran notas que se refirieran a célu- 
las madre y genética en general, cambio climático, nanotecnología, biodiversidad, influenza H1N1, recursos hídricos y biocombustibles, independientemente de que éstas bicieran referencia a los criterios mencionados arriba (es decir, que las noticias se basaran en resultados de investigación científica, consultaran explícitamente a investigadores o instituciones de investigación, etc). Esta salvedad temática, se constituye en una de las principales grietas que deja abierto el protocolo para que la ciencia y la tecnología, como articuladora de lo social, y no como mera práctica investigativa aislada de ello, se cuele dentro de la muestra y permita otras posibles lecturas, "menos limpias", sobre la presencia de estos temas en los medios masivos de comunicación.

Para abordar el análisis sobre aquello que se cuela dentro de la muestra, que como veremos en el apartado siguiente es lo que principalmente constituye el corpus de noticias identificado, buscamos combinar el análisis de contenido de carácter cuantitativo en que se basa el protocolo, con el análisis cualitativo, especialmente el de tipo semiodiscursivo (KRIPPENDORF, 1990). Así, nos detuvimos en los contextos de producción de sentido de aquellas notas que se identificaron por criterio temático y no sólo por cumplir con criterios propios y comunes del periodismo científico (desde la perspectiva de darle cierta preeminencia a los hallazgos, en el sentido de resultados de investigaciones y, en general, avances recientes en ciencia y tecnología). De manera particular en las notas inscritas en la categoría de medio ambiente y que fueron seleccionadas por estar relacionadas con cambio climático y con recursos hídricos, dos de las temáticas definidas en el protocolo como exentas de los requisitos del resto de temas, como lo acabamos de mencionar. Nuestra pregunta guía fue ¿qué contextos globales y locales enmarcan la emergencia de estas notas con más fuerza en el período de estudio?, ¿cómo se relaciona esto con ciertos sentidos tácitos de ciencia y tecnología?

Vale la pena anotar que desde este tipo de preguntas, la aparición de puntos ciegos (HARAWAY, 1995) da cuenta de las inferencias que se desprenden de la interacción entre los datos y el contexto, algo indispensable en cualquier análisis de contenido, como lo expone Krippendorf (1990): al fin y al cabo, dicho análisis es un método para estudiar el significado simbólico de mensajes a partir de lo que dicen los datos, pero también, claro está, de lo que no dicen.

\section{RESULTADOS}

Tomando como base el protocolo de análisis ya descrito, pasaremos ahora a hacer una descripción general de las tendencias encontradas en la cobertura de las noticias para luego adentrarnos en un análisis crítico de dos aspectos particulares de este protocolo. Los ítems que tomaremos como base para esa descripción general son: la distribución mensual y semanal, la duración, las características de las noticias en relación con el noticiero en su conjunto, y la forma en que son presentados los temas (en especial las fuentes, las voces y los encuadres).

En las 42 emisiones que fueron analizadas se encontraron un total de 
61 noticias relacionadas con temas de ciencia y tecnología. De ellas casi el 51\% correspondió a temas ambientales diversos relacionados principalmente con recursos hídricos $(34 \%)$, biodiversidad (8\%), cambio climático (8\%). Llama la atención que estos temas, por su parte, fuesen principalmente enmarcados en un contexto de daños y riesgos, antes que desde una perspectiva que resaltara las promesas y beneficios de la ciencia y la tecnología en sí. Sobre estos aspectos profundizaremos más adelante. Ahora bien, estas 61 noticias estuvieron distribuidas mensualmente de la siguiente manera:

\section{Gráfica 1: Piezas por mes}

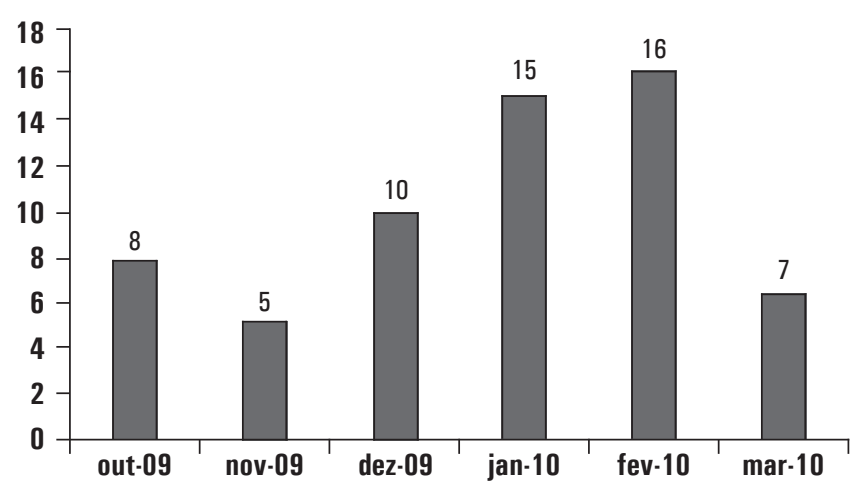

Como podemos notar se encontraron más noticias en el primer trimestre del $2010(62 \%)$ que en el último del $2009(38 \%)^{7}$. Considerando que se tomaron 6 semanas construidas para definir la muestra, los días en que más noticias se encontraron fueron los viernes, miércoles y lunes (gráfica 2). Con respecto a la duración de las noticias encontramos que el 77\% de la muestra tiene una duración inferior a los 2 minutos.

\section{Gráfica 2: Dia de la semana}

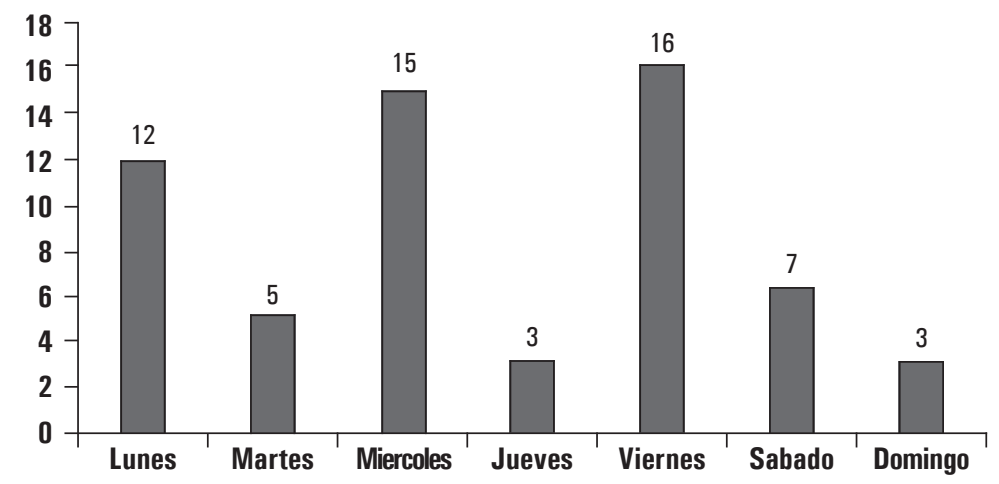


En relación con las características generales de las noticias en el marco del noticiero, llama la atención que el $50 \%$ de éstas se ubican en la parte final ${ }^{8}$ y no en el bloque inicial (17\%) o intermedio (33\%), así mismo encontramos que en su mayoría las noticias no son presentadas por medio de avances en la apertura del noticiero $(60 \%)$ y que tampoco hacen parte de series de piezas $(79 \%)$, es decir de notas sobre una misma noticia en varias emisiones. Estas características podrían ser indicativas de la poca relevancia que se le da a estos temas en los noticieros.

A propósito de la forma como se presentan las notas seleccionadas, encontramos que en la mayoría de los casos se alude a miembros del gobierno -mucho más que a científicos o académicos-, hombres en su mayoría, que fungen como fuentes o voces sobre el tema. Además, los ciudadanos aparecen como la segunda voz más importante. Esto concuerda con el principal encuadre que se les da a estas temáticas, que como se puede notar en la gráfica 3, está dado por la personalización (el encuadre que se centra en la narrativa personal y en el testimonio). Lo anterior dialoga con los resultados de los estudios presentados en los antecedentes y con lo que veremos más adelante.

\section{Gráfica 3: Encuadre}

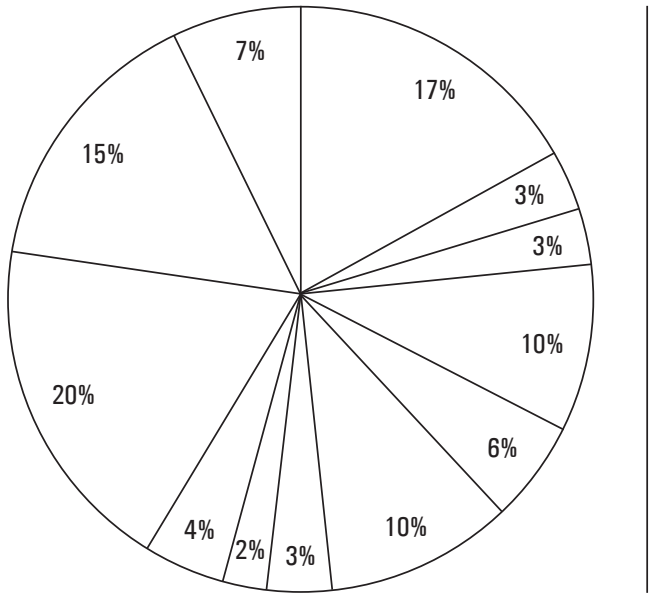

17\%: Nueva Investigación 3\%: Nuevo Desarrollo Tecnológico 3\%: Antecedentes Científicos 10\%: Impacto Cyt 6\%: Ética/moral 10\%: Estrategia Política, políticas públicas, regulación 3\%: Mercado, promesa económica, patentes, derechos propiedad 2\%: Controversia científica 4\%: Incertidumbre científica 20\%: Personalización 15: Cultural 7\%: Ninguno de los anteriores

Ahora bien, hay que subrayar que los científicos no son vistos como las principales fuentes de estas noticias, dado que las temáticas que se presentan en algunas ocasiones son noticias que refieren no directamente a desarrollos científicos sino a problemáticas más amplias (temas ambientales por ejemplo)?

\section{ANÁLISIS DE LOS RESULTADOS}

Como se explicó anteriormente, es importante dar cuenta de los resultados de este análisis de contenido a partir de lo que arrojan los datos explícita- 
mente, pero también de lo que queda tácito. Ahora bien, hacer inferencias que le sirvan al análisis requiere una mirada atenta al contexto y a las posibilidades que dan los criterios metodológicos, al mismo tiempo que a las limitaciones que dichos criterios implican. El hecho de darle prioridad a unos temas frente a otros, de registrarlos independientemente del abordaje que tengan, y el hecho de registrar los temas científicos tratados como "hallazgos científicos", puede generar algunos puntos ciegos que merecen atención. Esto, como se discutirá, se evidencia en los temas relacionados con los riesgos y con el medio ambiente.

En primer lugar, vale la pena anotar que, en términos generales, medio ambiente y salud suelen ser los temas en los que el público a menudo se siente más concernido por el papel de la ciencia y la tecnología en la sociedad; esto en el ámbito colombiano se puede ver en estudios de percepciones del público como el de OCyT (2009). Por su parte, el estudio de percepciones que se expone en Bonilla y Cadavid (2004) sobre los medios masivos tradicionales en Colombia, muestra que el tema de la salud -sumado a la educación- es el más demandado por los ciudadanos a los medios ${ }^{10}$. Lo anterior lo incorporan los medios en su contrato de lectura (VERÓN, 1983) o contrato mediático (DE CHEVEIGNÉ, 2000) con el público: es decir, el manejo que hacen los medios de los temas, desde el punto de vista del contenido y de la forma, se basa en un destinatario inscrito, en la coincidencia de imaginarios entre el medio y su público. En el caso particular de los datos arrojados por nuestro estudio, estos dos "macro-temas", medio ambiente y salud, son un reflejo de lo anterior, pues suman por lo menos 41 noticias -de las que sólo de recursos hídricos son 21-, es decir el 67.2\% del total ${ }^{11}$, como se puede observar en la gráfica 4.

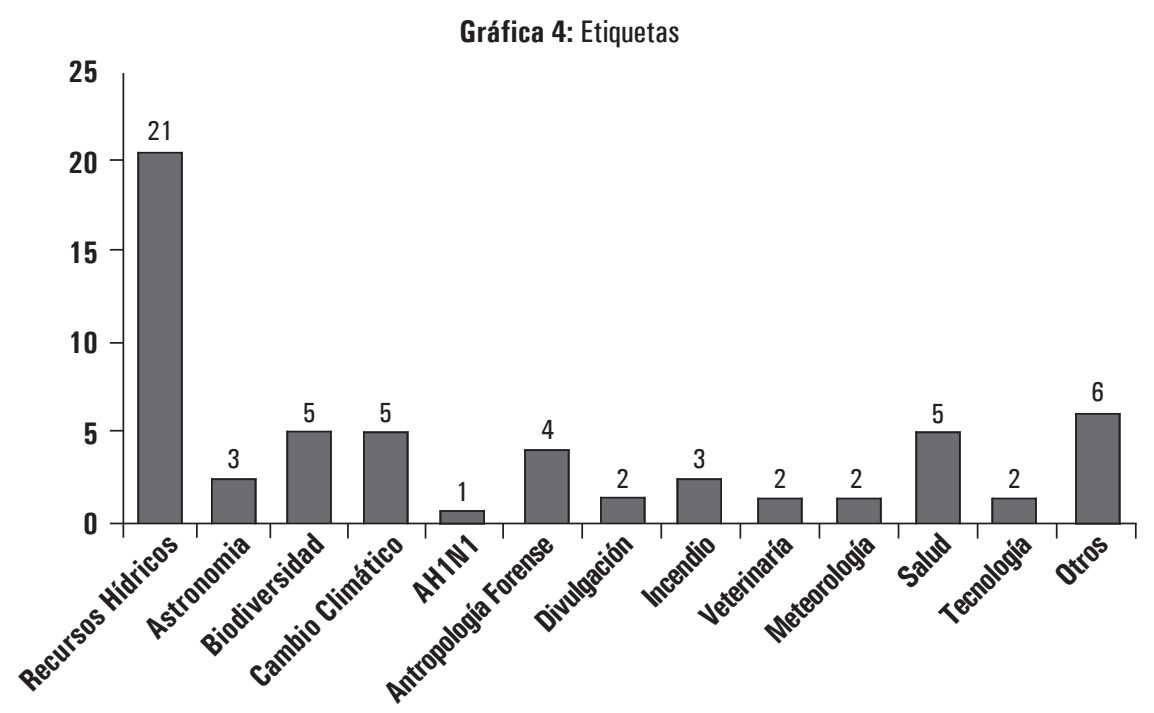

El hecho de que las notas relacionadas con el tema de los recursos hídricos constituyan el $34.4 \%$ del total, merece tratarse con detenimiento. Pero antes de eso, es preciso mencionar dos posibles puntos ciegos que se generaron a partir 
de las decisiones en la elaboración del protocolo metodológico para el registro de datos, y que tienen que ver con el macro-tema del medio ambiente. El primero es que el protocolo no incluye explícitamente un tema como el de los desastres naturales; esto, para un periodo como el de octubre de 2009 a abril de 2010 implica que no hubieran entrando en la muestra grandes noticias de relevancia internacional y, en especial, en Latinoamérica y el Caribe, como el sismo de Haití en enero de 2010 y el de Chile en febrero de 2010, sucesos que evidencian articulaciones estrechas entre ciencia, tecnología y sociedad. Con esto, las notas habrían sobrepasado, de lejos seguramente, las 61 en total encontradas en Noticias Caracol en dicho periodo.

Ahora bien, muchas noticias relacionadas con desastres naturales no se tratan periodísticamente como "hallazgos científicos", y menos con los criterios definidos en el protocolo usado en nuestro registro. No obstante, un tema como este suele utilizar información propia de la divulgación científica, tanto en los discursos como en las imágenes; esto nos lleva a pensar que podría haberse incluido en los temas a los que se les dio prioridad, independientemente de su abordaje, dada la importancia que tienen dentro del contexto científico mundial y, en particular, el latinoamericano ${ }^{12}$. Cabe agregar que dentro de las 21 notas de recursos hídricos de nuestra muestra, 7 tienen que ver con desastres naturales por lluvias y fenómenos climáticos, en Colombia y en el mundo; y 12 tienen que ver con la sequía generada por el fenómeno de El Niño, como se discutirá más adelante.

Otro posible punto ciego tiene que ver con la selección misma de la muestra. Como se dijo, en nuestro trabajo se utilizó el procedimiento denominado "semana construida", que es común y eficaz en estudios de esta naturaleza. Sin embargo, es evidente que un método de esta índole no permite hacerle seguimiento a cierto tipo de acontecimientos, y en especial a acontecimientos predecibles. Es el caso de la Cumbre Mundial sobre Cambio Climático celebrada en Copenhague, en diciembre 2009 que fue ampliamente cubierta por el informativo Noticias Caracol de las 7pm. Estos factores de contexto hay que tenerlos en cuenta, como se ve en Massarani y Buys (2007), para hacer las inferencias que comporta el análisis; y podría invitar a hacer muestreos de otra índole, como se expone en Krippendorff (1990). Hay que subrayar que de las 5 notas en total que hay sobre cambio climático, 3 estuvieron relacionadas con la cumbre y aparecieron durante el mes de diciembre.

Pero volvamos al tema que más apareció en la muestra y veamos la confrontación con otros datos. Las notas sobre el tema recursos hidricos no sólo suman el 34.4\% de la muestra, también cuadruplican a los temas que les siguen con mayor número de notas como cambio climático y biodiversidad. Si miramos los otros resultados, encontramos que hay una alta cantidad de noticias clasificadas en el área de ciencias ambientales, casi el 50\%, lo que es coherente con lo anterior. Hay también otros datos que son llamativos. En primer lugar, en la dimensión "tratamiento" definida en el protocolo, se hace énfasis en las consecuencias y efectos, con variables como daños, riesgos y recomendaciones. Estas tres últimas variables suman 76 "tratamientos" de este tipo, mientras que las variables promesas y beneficios suman solo $16^{13}$. Este es un indicador fuerte de que nos encontramos frente a una agenda 
mediática en la que las percepciones sobre el riesgo cobran bastante relevancia, lo que concuerda con que los temas más recurrentes tienen que ver con el medio ambiente, además de la salud, y con las demandas más probables del público en relación con la ciencia y la tecnología: algo que se ve desde una perspectiva que no se centra en los hallazgos en ciencia y tecnología.

En este punto vale la pena traer algunos elementos de contexto para continuar interpretando los datos. Uno muy importante tiene que ver con que durante la segunda mitad de 2009 y el primer semestre de 2010, arreció el denominado fenómeno de El Niño en Colombia ${ }^{14}$, con una sequía que tuvo grandes efectos económicos y sociales. Los resultados que se mencionaron antes sobre los temas, área y tratamientos se pueden explicar desde la importancia que este fenómeno tuvo en la muestra. Además, si se considera el uso de la técnica de semana construida, puede decirse que estamos frente a un acontecimiento que está presente en todo el corpus; algo consistente con que el tema recursos hídricos haya tenido tanta preponderancia. Veamos los datos de forma más precisa: de las 21 noticias sobre recursos hídricos, 12 tuvieron que ver con el fenómeno de El Niño y sus consecuencias (la ausencia de recursos hídrico principalmente). Es decir, que el $19.7 \%$ de la muestra estuvo dedicado a este tema en particular.

La injerencia de un fenómeno de este tipo en el informativo Noticias Caracol durante este periodo también se puede leer a la luz de otros datos como las fuentes y las voces. El dato más alto en las fuentes corresponde a miembros del gobierno, que duplica a la fuente que le sigue: cientificos, profesores e investigadores, en el total de la muestra. En el caso de recursos hídricos, la fuente miembros del gobierno aparece 10 veces, seguida por las fuentes ciudadanos y miembros del público, y representantes de la industria comercio, productores, que aparecen 2 veces cada una. En cuanto a las voces, las principales en el total de la muestra son: miembros del gobierno, $26.7 \%$, ciudadanos y miembros del público, 18.7\%, y expertos y profesionales especializados, 13.3\% . Para recursos hídricos la voz más frecuente es miembros del gobierno: 9 veces, el mismo número de voces de ciudadanos y miembros del público. Es decir, los miembros del gobierno son las fuentes más consultadas y las voces más representadas, algo que encaja con una preocupación de las autoridades frente a un fenómeno como el de El Niño, y también frente a los desastres generados por lluvias (el segundo ítem más importante dentro de las notas clasificadas como recursos hídricos, según se explicó antes). Lo anterior parece previsible: al fin y al cabo las autoridades gubernamentales son las primeras llamadas a responder ante las consecuencias de estos fenómenos.

Por su parte, el dato de los ciudadanos y miembros del público como la segunda voz más importante en toda la muestra, e igualmente importante a miembros del gobierno en lo que respecta a recursos hídricos, se acerca a los resultados del "encuadre": en este último el dato más alto es el de personalización, que se refiere a la narrativa personal de los testigos, y que constituye el $20 \%$ en el total de la muestra (como se puede observar en la gráfica 3); además es el tipo de encuadre más usado en recursos hídricos, y duplica al que le sigue: estrategia política, políticas 
públicas, regulación. Este último punto se puede contrastar con la relativa baja controversia que presenta el informativo en las notas analizadas (30\%), y dentro del tipo de controversia, la que "excede lo científico" da muy por encima (83\%) de la "controversia científica" $(17 \%)^{15}$; en el caso específico de recursos hídricos, todas las controversias exceden lo científico. Lo anterior nos muestra de nuevo que estamos frente a problemáticas que tocan directamente a los ciudadanos, como es el caso de los temas ambientales y en particular los ligados a los recursos hídricos y a las consecuencias del fenómeno de ElNiño ${ }^{16}$.

En suma, podemos ver que para un corpus basado en las emisiones de un informativo como Noticias Caracol, no siempre es pertinente el uso de variables clásicas de las investigaciones sobre los cubrimientos del periodismo científico, variables que se centren en los hallazgos, en los resultados de investigaciones, y en abordajes de los temas de ciencia y tecnología tan propios de los países industrializados. En nuestro caso, los temas registrados independientemente del abordaje-según los criterios de inclusión (exclusión) utilizados en la muestra-, en especial el de recursos hídricos, dieron lugar a la mayor parte de los datos y a las herramientas para correlacionarlos. Se ve entonces la necesidad de explorar los puntos ciegos y las líneas de fuga, con la ayuda de elementos de contexto, incluso exógenas a los registros, según las circunstancias. Por lo demás, la relevancia que tienen temas como los ambientales en las relaciones entre los medios -en especial los telediarios- y los públicos en Colombia, y las particularidades de dichos temas, invitan a hacer replanteamientos en las adaptaciones de metodologías desarrolladas en dichos países industrializados, para el estudio de nuestras realidades mediáticas en relación con la ciencia y la tecnología.

\section{SOBRE CONSIDERACIONES FUTURAS}

Quisiéramos cerrar este artículo aludiendo a aspectos que a nuestro parecer deberían tenerse en cuenta para estudios posteriores sobre la cobertura de la ciencia y la tecnología en los medios en países periféricos. En primer lugar hay que señalar que si bien el protocolo utilizado está pensado para un análisis de contenido de carácter principalmente cuantitativo -como ha sido la tendencia histórica de este enfoque-, sería oportuno considerar el papel que jugó en su diseño, y que puede jugar en estudios futuros, la combinación con el análisis cualitativo, especialmente el de tipo semiodiscursivo. Para esto basta detenerse en las variables aplicadas en la dimensión narrativa y sus encuadres (por ejemplo, si la noticia habla en términos de beneficios, promesas, daños, riesgos, etc.), y en la dimensión tratamiento, que están dedicadas a rastrear aspectos importantes del discurso, el uso de sonidos, y las formas y contenidos de las imágenes. Los avances en los manejos de los encuadres (frames) en el análisis de contenido, dan pistas en este sentido ${ }^{17}$.

Ahora bien, las posibilidades de combinaciones futuras con el análisis semiodiscursivo, allende protocolos como el que usamos en nuestro estudio, pueden ser 
bastante provechosas, como se puede observar en trabajos como el de De Cheveigné (2000). De hecho este cruce de aproximaciones en el análisis de contenido se ha venido decantando en los últimos años, como lo discute Bonilla (2011b). Si bien los enfoques semiodiscursivos se pueden aplicar con más detalle a muestras en principio más pequeñas, habría que considerar las facilidades que brindan herramientas diseñadas para manejar muestras más grandes, como es el caso del programa Atlas Ti.

En este punto, no hay que olvidar la marcada relación que tiene buena parte del análisis de contenido en su origen con los análisis funcionalistas, como se desprende de Kripendorff (1990); además de la relación de estos últimos con los modelos deficitarios en la comunicación pública de la ciencia y la tecnología, como lo expone Hermelin (2011).

Los trabajos de sociología de los medios pueden ser también vetas importantes en un futuro, para conectarlos con los tipos de análisis de contenido como el que nos concierne en este trabajo. Ahí podrían ser relevantes trabajos como el de Bonilla y Cadavid (2004), de nuevo, y el de Rey (2008). También habría que buscar conexiones entre los análisis de los mensajes, de contenido y semiodiscursivos, con los estudios de recepción y de percepciones. Aquí vale la pena referirse a trabajos clásicos como los que aparecen en Morley (1996), y tener en presente la importancia de los avances en los estudios de recepción en el contexto latinoamericano (JACKS, 2011) y colombiano (BONILLA, 2011a).

En este sentido este artículo ha buscado señalar una serie de desafíos para las metodologías de investigación sobre cubrimientos mediáticos de la ciencia y la tecnología en nuestros ámbitos; desafíos que permitan la adaptación de enfoques a nuestros contextos socioculturales, y que contribuyan a analizar lo explícito y a vislumbrar lo tácito sobre las maneras de entender el conocimiento por parte de los científicos y expertos, de los medios y de la sociedad; desafíos que incluyan cruces de diversas aproximaciones de los estudios de la comunicación y de otros campos. Con esto no sólo se busca responder a la naturaleza compleja de los objetos de estudio: también a la necesidad de que nuestros propios análisis puedan ser más reflexivos.

\section{NOTAS}

1 Los autores están listados en orden alfabético. Este artículo surge en el marco del proyecto de investigación "Ciencia y medios: un estudio comparativo de la cobertura de la ciencia en los noticieros de televisión de Brasil y Colombia”, realizado entre 2010 y 2011 y financiado por la Pontificia Universidad Javeriana (Bogotá, Colombia), la Universidad EAFIT (Medellín, Colombia) y la Fundação Oswaldo Cruz / Casa de Oswaldo Cruz / Museu da Vida, Río de Janeiro (Brasil). Además este artículo y los resultados del proyecto están vinculados a la Red Iberoamericana de Monitoreo y Capacitación en Periodismo Científico (www.museudavida.fiocruz.br/redejc), liderada por el Museu Da Vida, compuesta por grupos de investigación de 10 países, que cuenta con el apoyo del Programa Iberoamericano de Ciencia y Tecnología para el Desarrollo y del Conselho Nacional de Desenvolvimento Científico e Tecnológico (CNPq, de Brasil).

\footnotetext{
${ }^{2}$ En Colombia se usa el término "noticiero" para lo que en otros países denominan "telediario".
} 
${ }^{3}$ Como veremos más adelante, estas conclusiones son similares a algunas a las que se llega en el estudio que soporta la investigación aquí presentada.

${ }^{4}$ El diario nacional de mayor consumo en Colombia.

${ }^{5}$ Los criterios para la escogencia de este telediario fueron definidos por la Red Iberoamericana de Monitoreo y Capacitación en Periodismo Cientifico, e incluyeron el hecho de que este telediario ha mantenido un alto índice de audiencia; es un programa visto por distintos segmentos socioeconómicos de la sociedad colombiana, como parte de su cotidiano; es transmitido en horarios de mayor audiencia; y de vez en cuando cubre temas de ciencia y tecnología. Hay que señalar que en Colombia se considera que a las $7 \mathrm{pm}$ empieza la franja prime time (o Triple $A)$.

${ }^{6}$ Nos corresponde señalar que a este tipo de premisas quizás subyace, en muchos casos, un uso no deliberado del modelo deficitario en el trasfondo del análisis, que conlleva suponer que este tipo de espacios define algunos temas como prioritarios, sin dejar muy claro su sustento. Modelo del que a menudo es más fácil librarse en las reflexiones teóricas que en la práctica (Daza y Arboleda, 2007).

${ }^{7}$ Debe tenerse presente que las dos últimas semanas de diciembre (sobre todo la última) y las dos primeras semanas de enero (sobre todo la primera) son atípicas en cuanto a los temas y los tratamientos de las noticias en Colombia. Esto considerando que son las fiestas de navidad y de fin de año, y a menudo las noticias están marcadas por la carencia de acontecimientos, por ejemplo los que tienen que ver con las actividades de los políticos.

${ }^{8}$ Lo que las hace más cercanas en su ubicación a las secciones de deportes y de farándula (algo similar a lo que se denomina como Showbiss). Estas dos secciones son con las que suele cerrar Noticias Caracol y otros telediarios de canales de señal abierta en Colombia.

${ }^{9}$ En relación con este punto cabe anotar que a pesar de que algunas noticias hacen referencia a situaciones controversiales (30\%), de estas la mayoría (83\%) exceden el campo de lo científico, esto está señalado con el hecho de que las mismas fueron incluidas principalmente por criterios temáticos y no por que respondieran a los criterios de inclusión/exclusión ya mencionados, que privilegiaban noticias que refirieran a resultados de investigaciones científicas.

${ }^{10}$ Algo que contrasta en el mismo estudio con la posición que tienen los temas de ciencia, salud y medio ambiente y su relativa poca prioridad en la agenda de los medios, como se mencionó anteriormente.

${ }^{11}$ Recursos hídricos (21), cambio climático (5), biodiversidad (5), incendios (3) meteorología (2), para medio ambiente. Y en salud (5) sumado a H1N1 (1) (esto sin incluir antropología forense ni veterinaria).

${ }^{12} \mathrm{Si}$ aceptamos que hoy en día estamos en las denominadas "sociedades del riesgo" -según la expresión de Beck (2006)-, lo que en buena partese debe a las interacciones tan estrechas entre ciencia, tecnología, sociedad y medio ambiente, es entonces necesario que para estudios de este tipo consideremos la categoría desastres naturales como algo por lo que deba indagarse.

${ }^{13}$ Esta dimensión de tratamiento utilizaba sólo estas 5 categorías: daños, riesgos, recomendaciones, promesas y beneficios.

${ }^{14}$ En este sitio web oficial del gobierno de Estados Unidos, se pueden ver datos al respecto. Aparecen en términos del nombre científico: el fenómeno Enso, que incluye dos estados, El Niño y La Niña: http://www.cpc.ncep.noaa.gov/products/analysis_monitoring/ensostuff/ensoyears.shtml (visitado el 28 de junio de 2011). 
La cobertura de la ciencia en los noticieros colombianos: del análisis de resultados a las reflexiones metodológicas para su investigación.

${ }^{15}$ Nos referimos a la que se da sobre hechos científicos, teorías y paradigmas, entre otros, normalmente en el seno de la comunidad científica.

${ }^{16}$ Esto nos invita a hacer replanteamientos aspectos como: ¿qué tanto estamos dando lugar en los estudios de esta naturaleza a un distanciamiento de modelos deficitarios y difusionistas?, ¿acaso dichos modelos siguen tácitos en nuestros estudios sobre cubrimientos de temas de ciencia y tecnología en los medios?, y ¿estamos manteniendo una lógica de esfera pública ilustrada y tradicional, o estamos incluyendo en nuestros estudios la lógica de esfera pública ampliada, con sus múltiples racionalidades, y con los intereses de la sociedad civil y de los públicos?

${ }^{17}$ Ejemplo de esto es el estudio que se realizó con base en esta misma muestra en relación con la genética de poblaciones y que buscó identificar cómo la manera como estas noticias ratificaban un lugar del conocimiento científico como testigo modesto de ciertas políticas raciales y de seguridad democrática. Ver Díaz del Castillo, Olarte Sierra y Pérez Bustos, (2012).

\section{REFERENCIAS BIBLIOGRÁFICAS}

ALTHEIDE, D. Ethnographic content analysis. Qualitative Sociology, v. 10, n. 1, p. 65-77, 1985

AMORIM, L., MASSARANI, L. Periodismo científico: un estudio de caso de tres periódicos brasileros. R.B.E.C.T., v. 1, n.1, jan/abr, 2008.

BAUER, M., RAGNARSDÓTTIR, Á., RÚDÓLFSDÓTTIR A. science and technology in the british press, 1946-1990 - a systematics content analysis of the press, work report, 1993.

BECK, U. La sociedad del riesgo. Hacia una nueva modernidad. Barcelona: Paidós., 2006.

BONILLA, J. I., CADAVID BRINGE, A. ¿Qué es noticia? Agendas, periodistas y ciudadanos. Bogotá: Pontificia Universidad Javeriana - Fundación Konrad Adenauer, 2004.

BONILLA J. I. Re-visitando los estudios de recepción-audiencias en Colombia. Una revisión crítica, un debate necesario. Comunicación y sociedad., n. 16. p. 75-103, 2011a

El análisis de contenido. Definiciones, trayectos y consideraciones metodológicas, Producto inédito, 2011b.

BUSTOS MORA, G. Ciencia y tecnología en la prensa costarricense: análisis de casos. Revista de Ciencias Sociales, Universidad de Costa Rica, n. 106-107, p. 215-229, 2004.

CLARK, F., ILLMAN, D. A Longitudinal Study of the New York Times Science Times Section. Science Communication, v. 27, n. 4, p. 496-513, 2006.

DAZA, S., ARBOLEDA, T. Comunicación pública de la ciencia y la tecnología en Colombia: ¿políticas para la democratización del conocimiento? Signo y pensamiento, v. 25, n. 50, p. 101-125, 2007.

DE CHEVEIGNE, S. L'environnement dans les journaux télévisés. Médiateurs et visions du monde. París: CNRS Éditions, 2000.

DIAZ DEL CASTILLO, A., OLARTE SIERRA, M., PÉREZ BUSTOS, T. Testigos modestos y poblaciones invisibles en la cobertura de la genética humana en los medios de comunicación colombianos. Interface, Comunicação, Saude, Educação, (en prensa), 2012.

HARAWAY, D. Conocimientos situados: la cuestión científica en el feminismo y el privilegio de la perspectiva parcial. In Ciencia, cyborgs y mujeres. La reinvención de la naturaleza, Feminismos. Madrid: Ediciones Cátedra, 1995, p. 313-346

HERMELIN, D. Un contexto para la comunicación pública de la ciencia y la tecnología en Colombia: de las herencias eurocéntricas a los modelos para la acción. Co-herencia, n. 14, p. 231-260, 2011. JACKS, N. Análisis de recepción en América Latina. Un recuento bistórico para el futuro. Quito: CIESPAL, 2011.

KRIPPENDORFF, K. Metodología de análisis de contenido. Teoría y Práctica. Barcelona: Paidós, 1990.

MASSARANNI, L., BUYS, B. Science in the press in nine Latin American countries. Brazilian Journalism Research, v.3, n.2, p.77-96, semestre 2, 2007. 
MASSARANNI, L., BUYS, B.. "Cuando la ciencia es noticia: una evaluación de la sección de ciencia en nueve países de América Latina y el Caribe”. In: LOZANO, Mónica., SÁNCHEZ-MORA, Carmen (Ed.). Evaluando la comunicación de la ciencia: Una perspectiva latinoamericana, México D.F.: CYTED, AECI, DGDC-UNAM, 2008, p.115-128.

MASSARANI, L., AMORIM, L.E., BUYS, B., VENEU, F. Science Journalism in Latin America: A case study of seven newspapers in the region. Journal of Science Communication, v. 4, n. 3, 2005. Disponible en: http://jcom.sissa.it/archive/04/03/A040302/jcom0403\%282005\%29A02.pdf. Visitado el: 25 de julio de 2011

MASSARANNI, L., DAL COL, F., BUYS, B., ALMEIDA, C. A cobertura de ciência por jornais diarios: em pauta a pesquisa nacional na Argentina, no Brasil e no México. Razón y Palabra, México, año 13, n. 65, noviembre-diciembre 2008. Disponible en: http://www.razonypalabra.org.mx/N/ n65/actual/lmassarani.html. Visitado el: 25 julio 2011.

MORLEY, D. Televisión, audiencias y estudios culturales. Buenos Aires: Amorrortu, $1996 .$.

OBSERVATORIO COLOMBIANO DE CIENCIA Y TECNOLOGÍA. Percepciones sobre la ciencia y la tecnología en Bogotá. Bogotá: Observatorio Colombiano de Ciencia y Tecnología, 2009.

PARODI, G., FERRARI, S. El discurso de la ciencia y la tecnología en la prensa escrita chilena: una aproximación al corpus DICIPE-2004. D.E.L.T.A., v. 23, n. 2, p.339-369, 2007.

PELLECHIA, M. G. Trends in science coverage: A content analysis of three US newspapers. Public Understanding of Science, v. 6, n. 1, p. 49-68, 1997.

PETERS, H. P., BROSSARD, D., CHEVEIGNÉ, S., DUNWOODY, S., KALLFASS, M., MILLER, S., TSUCHIDA, S. Interactions with the mass media. Science, v. 321, n. 5886, p. 204-205, julio 2008. REY, G. La otra cara de la libertad. La responsabilidad social empresarial en medios de comunicación en América Latina. Bogotá: FNPI, Fundación Carolina, Pontificia Universidad Javeriana, Avina, 2008.

ROZO, C. Representaciones de cultura científica y cultura tecnológica desde los medios de comunicación. Diario El Tiempo, caso de estudio. I Congreso Iberoamericano de Ciencia, Tecnología, Sociedad e Innovación, México: OEI, 19 al 23 de Junio de 2006, p. 1-19.

STEMPEL, G.H., WESTLEY, B.H. (eds.). Research Methods in Mass Communication, Englewood Cliffs, NJ: Prentice Hall, 1989.

VERON, E.. Quand lire c'est faire: l'énonciation dans le discours de la presse écrite. Sémiotique, II. París: Institut de recherches et d'études publicitaires, 1984, p. 33-56.

Data de Recebimento: 02/04/2010

Data de Aprovação: 31/10/2010

Data da Versão Final: 03/11/2011 